\title{
Amperometric and Voltammetric Detection in Batch Injection Analysis
}

\author{
Christopher M. A. Brett, ${ }^{\circ \dagger}$ Ana Marla Olivelra Brett, ${ }^{\circ, \dagger}$ and Luclan Costel Miltoserlu ${ }^{\ddagger}$ \\ Departamento de Química, Universidade de Coimbra, 3049 Coimbra, Portugal, and Department of Biophysics, \\ University of Bucharest, Bucharest, Romania
}

Batch injection analysis (BIA) enables the investigation of electrode reactions using extremely small volumes of analyte solution of $100 \mu \mathrm{L}$. The potentialities of amperometric and voltammetric detection have been examined by studying the oxidation of ferrocyanide and ascorbic acid at platinum electrodes and using sample volumes between 10 and $100 \mu \mathrm{L}$. The electrolysis efficiency as a function of injected volume has been addressed. Consecutive injections during a slow linear potential sweep permit the registering of a point-by-point pseudo-steady-state voltammetric curve and allow the measurement of thermodynamic and kinetic parameters as well as the determination of concentration. Cyclic voltammetry during or after injection can aid in mechanistic studies. The combination of BIA and square-wave voltammetry leads to a continuous voltammetric profile from a single injection using $100 \mu \mathrm{L}$ : calibration plots show a factor of 4 sensitivity increase relative to fixed-potential amperometric detection.

Recent tendencies in electroanalysis have been toward the miniaturization of the electrode assemblies, the minimization of pretreatment procedures, and the ability to get the required information in the shortest time possible. The technique of batch injection analysis (BIA) ${ }^{1}$ permits the analysis of small volumes of analyte solution of less than $100 \mu \mathrm{L}$ with minimum sample pretreatment and has found a number of applications: electrochemical, e.g. ${ }^{2-5}$ thermometric ${ }^{6}$ and fluorometric. ${ }^{7}$ In electrochemical amperometric detection, a sample is injected from a micropipet tip directly over the center of a disk electrode held at constant applied potential and immersed in blank solution, which leads to the registering of an amperometric transient. The height of the transient as manifested by the maximum current leads directly to the concentration of electroactive species. Similarities of this system to the walljet system have been discussed elsewhere, as has the form of the chronoamperometric response. ${ }^{8}$

In this paper, the potentialities of the BIA technique for voltammetric and amperometric detection are further explored by analyzing the charge passed during the injection period

* To whom correspondence should be addressed. FAX: +351-39-27703

- Universidade de Coimbra.

University of Bucharest.

(1) Wang, J.; Taha, Z. Anal. Chem. 1991, 63, 1053-6.

(2) Chen, L.; Wang, J.; Angnes, L. Electroanalysis 1991, 3, 773-6.

(3) Wang, J.; Lu, J.; Chen, L. Anal. Chim. Acta 1992, 259, 123-8.

(4) Amine, A.; Kauffmann, J.-M.; Palleschi, G. Anal. Chim. Acta 1993, 273 213-8.

(5) Wang, J.; Taha, Z. Anal. Chim. Acta 1991, 252, 215-21.

(6) Wang, J. Taha, Z. Anal. Lett. 1991, 24, 1389-400.

(7) Wang, J.; Rayson, G. D.; Taha, Z. Appl. Spectrosc. 1992, 46, 107-10.

(8) Brett, C. M. A.; Oliveira Brett, A. M.; Costel-Mitoseriu, L. Electroanalysis, in press. and by considering the combination of BIA with linear sweep and cyclic voltammetry and with square-wave voltammetry.

\section{EXPERIMENTAL SECTION}

The cell for BIA measurements is a modified open wall-jet cell constructed of Perspex and filled with inert electrolyte. ${ }^{8}$ This cell fixes the micropipet tip (internal diameter, $a$, of 0.47 $\mathrm{mm}$ ) exactly over the center of a platinum disk electrode of radius $R_{1}=1.64 \mathrm{~mm}$, which was polished with diamond lapping spray down to a $1-\mu \mathrm{m}$ particle size before each series of experiments. The tip-electrode distance was optimized at 3 $\mathrm{mm}$. The cell also contains a platinum gauze counterelectrode and a saturated calomel reference electrode.

Injections were performed using a programmable motorized electronic micropipet (EDP Plus 100 EP-100, Rainin Instrument $\mathrm{Co}$. Inc.) that permits injections of between 10 and 100 $\mu \mathrm{L}$ in increments of $0.1 \mu \mathrm{L}$. Among the programming modes, single or consecutive injections, up to a total volume of 100 $\mu \mathrm{L}$, are possible at three different flow rates, which were calibrated as $24.5,47.6$, and $75.3 \mu \mathrm{L} \mathrm{s}^{-1}$.

A PC computer-controlled EG \& G PAR273A potentiostat was used for running the electrochemical experiments, with M270 Research Electrochemistry Software and M271 Cool Kinetic Analysis software.

Experiments were done using solutions prepared from analytical grade chemical reagents and Millipore Milli-Q ultrapure water at room temperature $\left(25 \pm 1^{\circ} \mathrm{C}\right)$. These were of $\mathrm{K}_{4} \mathrm{Fe}(\mathrm{CN})_{6}$ in $0.4 \mathrm{M} \mathrm{K}_{2} \mathrm{SO}_{4}$ electrolyte and ascorbic acid in $0.2 \mathrm{M}$ acetate buffer at $\mathrm{pH} 3.5$.

\section{RESULTS AND DISCUSSION}

Typical transients for the two-electron oxidation of 2.0 $\mathrm{mM}$ ascorbic acid are shown in Figure 1 for the three different flow rates, registered at $+0.8 \mathrm{~V}$ vs SCE to ensure that the limiting current plateau has been reached. A linear dependence on $V_{\mathrm{f}}^{3 / 4}$ is predicted from the equation for the limiting current $\left(I_{L, \mathrm{D}}\right)$ at the wall-jet disk electrode ${ }^{9}$

$$
I_{\mathrm{L}, \mathrm{D}}=1.43 n F R_{\mathrm{l}}{ }^{3 / 4} V_{\mathrm{f}}^{3 / 4} D^{2 / 3} v^{-5 / 12} a^{-1 / 2} c_{\infty}
$$

where $R_{1}$ is the disk electrode radius, $V_{\mathrm{f}}$ is the volume flow rate of solution, $D$ is the diffusion coefficient of the electroactive species of bulk concentration $c_{\infty}, \nu$ is the solution viscosity, and $a$ is the jet diameter. The data points in the plot of Figure

(9) Brett, C. M. A.; Oliveira Brett, A. M. Comprehensive Chemical Kinetics: Bamford, C. H., Compton, R. G., Eds.; Elsevier: Amsterdam, 1986; Vol. 26, Chapter 5 .

Analytical Chemistry, Vol. 66, No. 19, October 1, 1994

3145 


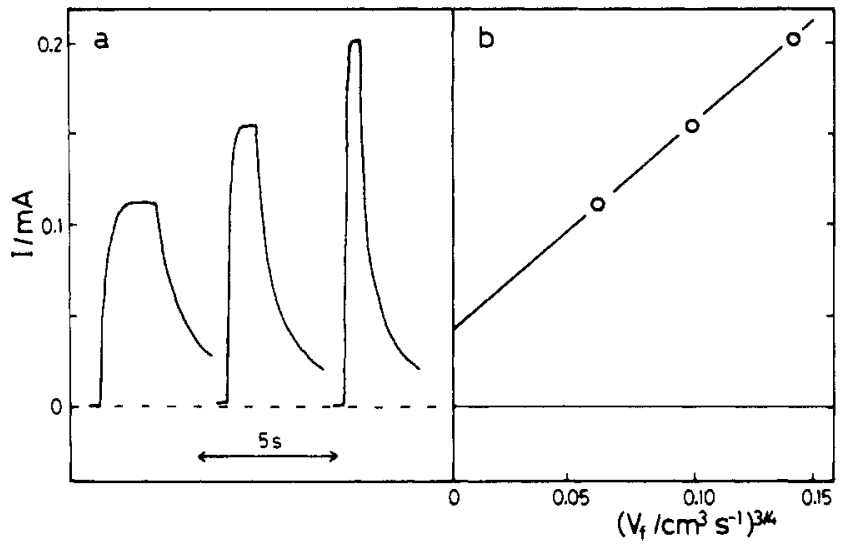

Figure 1. Oxidation of ascorbic acid (concentration $2.0 \mathrm{mM}$ ). (a) BIA transients recorded at $+0.8 \mathrm{~V}$ vs SCE at the three flow rates of 24.5, 47.6, and $75.3 \mu \mathrm{L} \mathrm{s}^{-1}$. (b) Plateau current vs $V_{f}^{3 / 4}$.

$1 \mathrm{~b}$ give a straight line but the relation in eq 1 is verified only at the highest of the three flow rates. There is a significant positive intercept in the plot, also found with ferrocyanide oxidation. ${ }^{8}$ This can be ascribed to the effects of radial diffusion which increase the expected currents at low flow rates in relation to those predicted by the theoretical equation, which does not take into account the contribution from radial diffusion..$^{10}$ As the flow rate is increased, this contribution becomes progressively less important. It was shown in ref 10 that the minimum flow rate for which radial diffusion effects begin to be negligible is around $50 \mu \mathrm{L} \mathrm{s}^{-1}$ for ferrocyanide oxidation, which corresponds to the medium flow rate in these BIA experiments. The currents obtained at the high flow rate are in reasonable agreement with the theoretical equation when we substitute $D=0.65 \times 10^{-5} \mathrm{~cm}^{2} \mathrm{~s}^{-1},{ }^{1} \nu=0.90 \times 10^{-2}$ $\mathrm{cm}^{2} \mathrm{~s}^{-1}$, and the geometrical parameters of the wall-jet cell.

From transients such as those shown, it was found that the minimum injectable volume of ascorbic acid necessary to reach the current plateau was around $20 \mu \mathrm{L}$, which should be contrasted with $14 \mu \mathrm{L}$ for ferrocyanide oxidation. This difference reflects the more complicated nature of the electrode process for ascorbic acid oxidation on polycrystalline platinum. ${ }^{11}$ The minimum volume was therefore fixed at $22 \mu \mathrm{L}$ in order to ensure that the current plateau was achieved. Thus, a maximum number of four consecutive injections could be performed, each of a maximum volume of $25 \mu \mathrm{L}$. This was used to test the repeatability (see Figure 2 ) the reproducibility (i.e., after dismantling and remounting the cell), and the conduction of other experiments. From these tests, a relative standard deviation of $0.3 \%$ of the bulk concentration was calculated in both cases, leading to a detection limit of $1.8 \times$ $10^{-5} \mathrm{M}$ for this simple amperometric technique. Transients recorded in the region of the detection limit validated this.

Calibration plots were constructed from data obtained using solutions of concentration varying from $2 \times 10^{-5} \mathrm{M}$ up to 2 $\mathrm{mM}$ of electroactive species; these were linear in all cases and with zero intercept. The results obtained for ferrocyanide oxidation were as follows for the three flow rates: $2.62 \pm 0.02$ $\times 10^{-2}, 3.86 \pm 0.03 \times 10^{-2}$, and $5.21 \pm 0.03 \times 10^{-2} \mu \mathrm{A} / \mu \mathrm{M}$. For ascorbic acid oxidation, the corresponding values were

(10) Brett, C. M. A.; Oliveira Brett, A. M.; Fisher, A. C.; Compton, R. G. J. Electroanal. Chem. 1992, 334, 57-64.

(11) Karabinas, P.; Jannakoudakis, D. J. Electroanal. Chem. 1984, 160, 159-67.

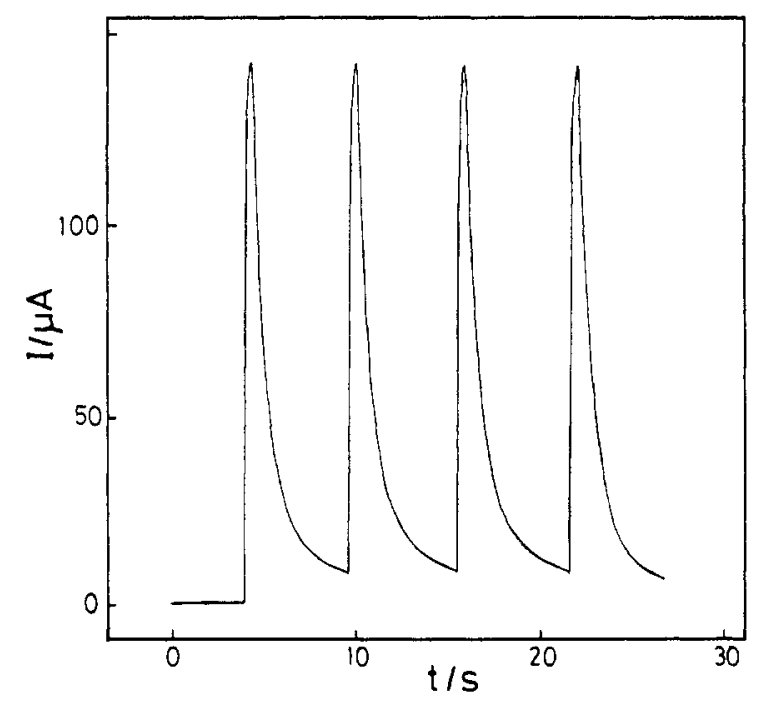

Figure 2. Repeatablity of ascorbic acid injection by BIA at a flow rate $47.6 \mu \mathrm{L} \mathrm{s}^{-1}$ ([ascorbic acid] $=2.0 \mathrm{mM}$; four injections of $25 \mu \mathrm{L}$ ).

$5.51 \pm 0.03 \times 10^{-2}, 7.45 \pm 0.03 \times 10^{-2}$, and $9.02 \pm 0.04 \times$ $10^{-2} \mu \mathrm{A} / \mu \mathrm{M}$.

Electrolysis Efficiency. An important parameter in the transients obtained is the electrolysis efficiency, $\eta$, defined as the charge passed divided by the charge corresponding to total electrolysis of the injected solution, $Q=n F V c_{\infty}$ where $V$ is the injected volume and $c_{\infty}$ is the bulk concentration of injected species. For large injection volumes, this would be expected to tend toward the current efficiency at the wall-jet electrode under steady-state conditions. Total conversion corresponds to

$$
I=n F V_{\mathrm{f}} c_{\infty}
$$

Combining this with eq 1 , the wall-jet current efficiency is therefore

$$
\eta(I)=1.39 R^{3 / 4} V_{f}^{1 / 4} D^{2 / 3} v^{-5 / 12} a^{-1 / 2}
$$

This was evaluated for the oxidation of ferrocyanide. Substituting values for potassium ferrocyanide in $0.4 \mathrm{M} \mathrm{K}_{2} \mathrm{SO}_{4}$ of $D=0.62 \times 10^{-5} \mathrm{~cm}^{2} \mathrm{~s}^{-1}$ and $\nu=0.94 \times 10^{-2} \mathrm{~cm}^{2} \mathrm{~s}^{-1},{ }^{9}$ we obtain a theoretical current efficiency of $0.7 \%$. The same efficiency is calculated for ascorbic acid oxidation.

Figure 3a is a schematic transient showing how the charge passed can be measured: either $Q^{\prime}$ up to the end of the current plateau $\left(t=t_{2}\right)$ or $Q^{\prime \prime}$ for $t=t_{2}+10 \mathrm{~s}$ when the current transient is effectively complete. In Figure $3 b$, typical $Q$ vs $t$ transients are shown for ascorbic acid oxidation at the three flow rates. $Q^{\prime}$ corresponds to the end of the linear portion of each charge transient. It can be seen that, apart from the slopes of the linear portions decreasing with decreasing flow rate, the total charge passed is higher the lower the flow rate. The differences between high and medium flow rates is not great, but the low flow rate leads to significantly higher $Q$ values. This is another manifestation of the effects of radial diffusion discussed in the previous section. Similar plots are obtained for other injected volumes and also for ferrocyanide oxidation. 


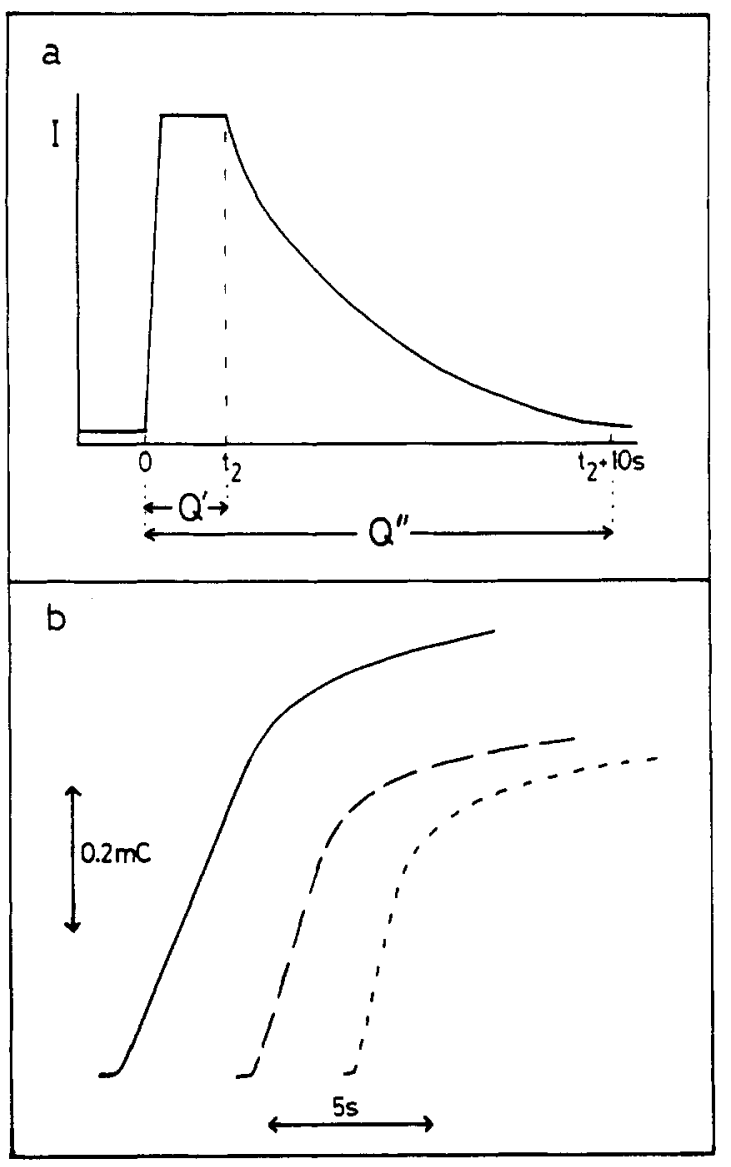

Flgure 3. (a) Schematic BIA translent showing the definition of $Q^{\prime}$ and $Q^{\prime \prime}$. (b) $Q$ vs $t$ plots for injection of $100 \mu \mathrm{L}$ of $2.0 \mathrm{mM}$ ascorbic acid at $0.8 \mathrm{~V}$ vs SCE at flow rates of $(--) 24.5,(--) 47.6$, and $(-) 75.3$ $\mu \mathrm{L} \mathrm{s}^{-1}$.

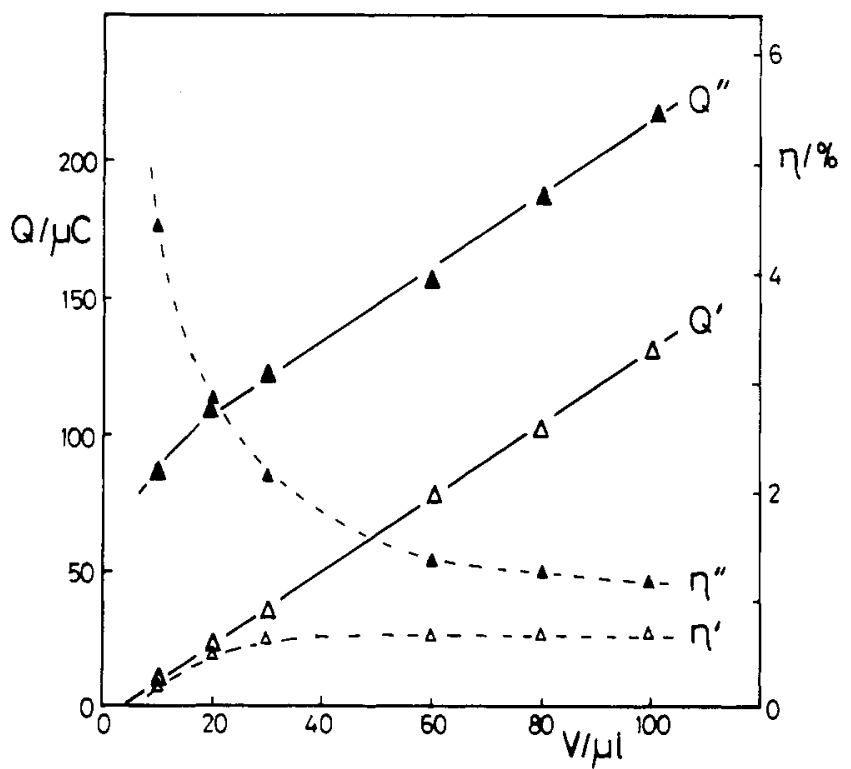

Figure 4. Values of $Q^{\prime}$ and $Q^{\prime \prime}$ (see Figure 3a) and respective electrolysis efficiencies, $\eta^{\prime}$ and $\eta^{\prime \prime}$, for BIA using the oxidation of ferrocyanide $\left(\left[\mathrm{K}_{4} \mathrm{Fe}(\mathrm{CN})_{\mathrm{B}}\right]=2.0 \mathrm{mM} ; E=+0.6 \mathrm{~V}\right.$ vs SCE$)$ at $75.3 \mu \mathrm{L} \mathrm{s} \mathrm{s}^{-1}$ flow rate.

We now bring together the $Q^{\prime}$ and $Q^{\prime \prime}$ values and calculate electrolysis efficiencies. Figure 4 shows the charge and calculated electrolysis efficiency as a function of volume from BIA experiments involving the oxidation of potassium ferrocyanide at the high flow rate where radial diffusion effects can be neglected. For large injection volumes approaching
$100 \mu \mathrm{L}$, the electrolysis efficiency corresponding to $t=0 \mathrm{up}$ to $t=t_{2}$ does indeed correspond to the wall-jet current efficiency dependence given by eq 3 since the contribution of the charge after the injection finishes $\left(t>t_{2}\right)$ becomes relatively minor. It can also be seen that the charge under the falling portion of the curve is relatively constant and leads to a dislocation of the charge curve by an approximately constant amount to more positive values. The faradaic charge efficiency also increases.

Two conclusions can be taken from these plots. First, although the variation of $\eta^{\prime}$ or $\eta^{\prime \prime}$ with volume is not linear, it may be that measurement of the charge under the peak rather than the maximum current for lower injection volumes leads to more favorable conditions for improving sensitivity. Secondly, the electrolysis efficiency is higher at low flow rates due to radial diffusion contributions, which may also aid in improving sensitivity.

BIA and Linear Sweep/Cyclic Voltammetry. The use of more sophisticated instrumentation techniques allows increased quantities of information to be obtained from one experiment. In particular, recording transients at different points on the voltammetric wave can lead to the possibility of obtaining thermodynamic and kinetic as well as analytical information.

Therefore, instead of doing several consecutive injections at fixed potential, and in order not to lose the benefits of the fast analysis time offered by BIA, they were performed during a slow linear potential sweep. Peaks were obtained at different potentials, corresponding to a point-by-point pseudo-steadystate voltammogram. This was done for both ferrocyanide and ascorbic acid oxidation. Typical results are shown in Figures 5 and 6 . The maximum number of consecutive injections is determined by the minimum volume necessary to reach the plateau current and is six for ferrocyanide and five for ascorbic acid, using $100 \mu \mathrm{L}$ of sample solution. Although there is incomplete "washout" between consecutive injections, i.e., the current does not return to zero, this has no effect on the absolute value of the maximum current registered during the next injection. This is because, due to the wall-jet hydrodynamics, the fluid reaching the electrode surface during an injection period comes purely from the injection itself; the solution from previous injections is pushed away radially.

Inspection of Figures 5a and 6a shows the characteristic shape of a steady-state voltammogram on joining the points of maximum current, and the parameters obtainable from steady-state voltammograms can be determined. Apart from the concentration determination in the limiting current region, the construction of mass-transport-corrected Tafel plots in the form of $\lg \left\{\left(I_{\mathrm{L}}-I\right) / I\right\}$ vs $E$, Figures $5 \mathrm{~b}$ and $6 \mathrm{~b}$, permits the calculation of the half-wave potential. These are 0.220 and $0.425 \mathrm{~V}$ vs SCE for ferrocyanide and ascorbic acid, respectively. The inverse slope of the Tafel plot is $68 \mathrm{mV}$ in the case of ferrocyanide (Figure $5 b$ ), which confirms the near-reversible one-electron oxidation process. For ascorbic acid (Figure 6b), the inverse slope is $250 \mathrm{mV}$, verified in many successive experiments, which leads to $\alpha n \sim 0.25$. Thus, on polycrystalline platinum at this $\mathrm{pH}$ of 3.5 , the process is much more complex and has been suggested to involve a preadsorption process; ${ }^{11}$ it is the object of further studies at the present. 


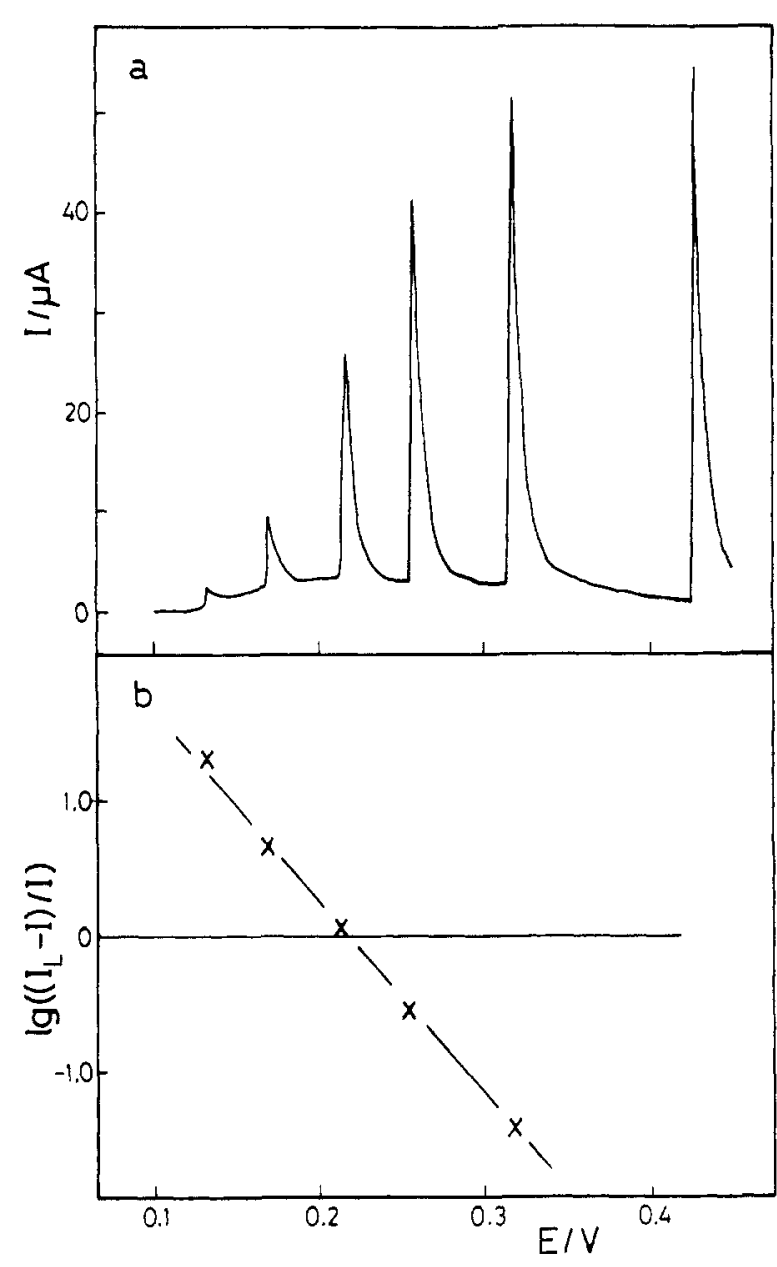

Figure 5. Pseudo-steady-state point-by-point voltammetric curves for ferrocyanide oxidation. (a) BIA transients for six consecuttve injections of $16 \mu \mathrm{L}$ of $2.0 \mathrm{mM}$ ferrocyanide during a linear potential sweep; scan rate, $10 \mathrm{mV} \mathrm{s}^{-1}$. (b) Corresponding mass-transport-corrected Tafel plot.

Further information may be obtained by performing cyclic voltammetry on the injected analyte sample: this can be done after or during the injection. For purely diagnostic purposes and at commonly employed scan rates (for example 50 or 100 $\mathrm{mV} \mathrm{s}^{-1}$ ), it is usually more easily performed after injection. This is because some of the injected analyte solution remains in the zone close to the electrode; however, its concentration will, in general and as verified by us, be slightly less than in the injected analyte due to mixing. So this method is not highly accurate for quantitative concentration determination. A typical result for ferrocyanide is demonstrated in Figure 7a. This shows all the characteristic features of the cyclic voltammogram, including the expected variation with scan rate.

Alternatively, a potential scan can be done during the injection period: wall-jet hydrodynamic effects arise at low scan rate as shown in Figure $7 \mathrm{~b}$ and documented elsewhere. ${ }^{12}$ At high scan rates, the hydrodynamic effects become small, and after blank subtraction, the same voltammogram as in the stationary solution is obtained (Figure 7). A minimum potential scan rate has to be calculated for the three flow rates and as a function of injected volume such that the full cycle occurs during the injection period.

(12) Compton, R. G.; Fisher, A. C.; Latham, M. H.; Brett, C. M. A.; Oliveira Brett, A. M. J. Phys. Chem. 1992, 96, 8363-7.

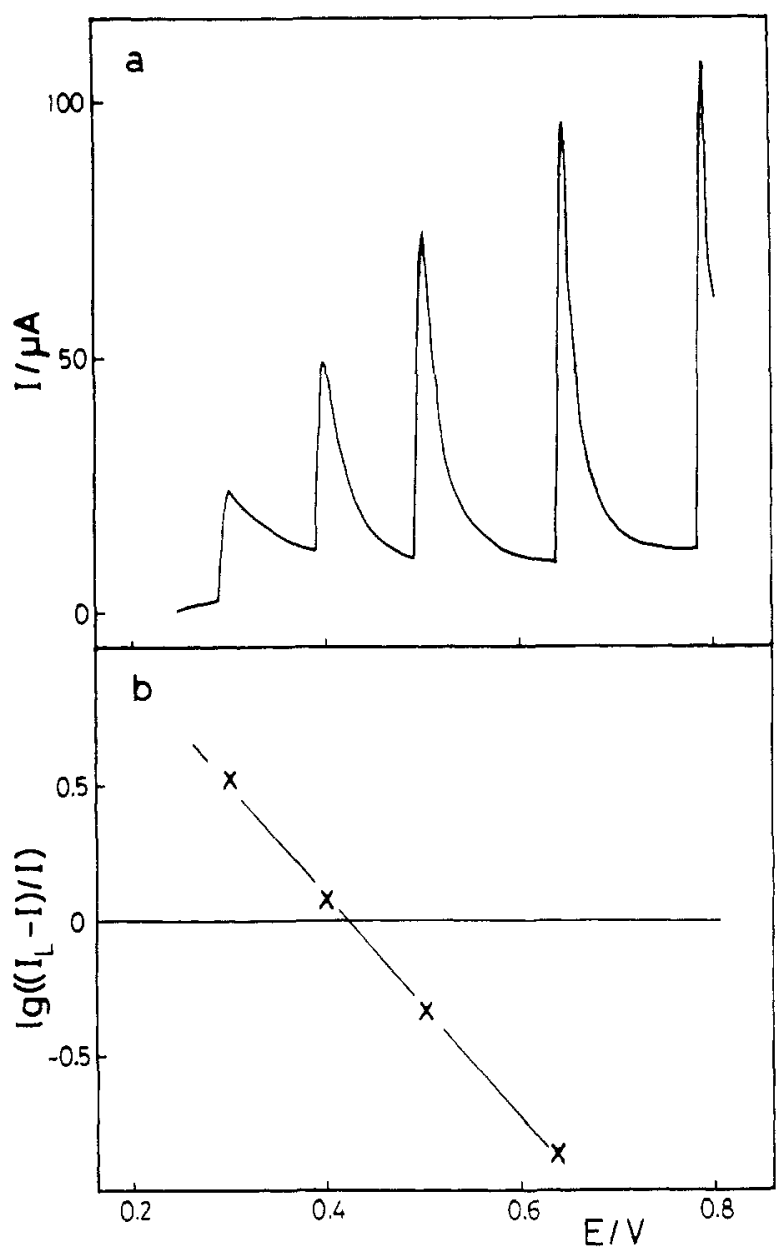

Flgure 6. Pseudo-steady-state point-by-point voltammetric curves for ascorbic acid oxidation. (a) BIA transients for five consecutive injections of $20 \mu \mathrm{L}$ of $2.0 \mathrm{mM}$ ascorbic acid during a linear potential sweep; scan rate, $10 \mathrm{mV} \mathrm{s}^{-1}$. (b) Corresponding mass-transport-corrected Tafel plot.

The implications of this method of analysis are far-reaching since $100 \mu \mathrm{L}$ or less of the sample solution is consumed. The advantages of BIA will become even more apparent with square-wave voltammetry.

BIA and Square-Wave Voltammetry. An alternative method for analyzing the voltammetric wave and for determining concentration, not at fixed points but continuously, is through the use of square-wave voltammetry. This technique offers the advantage of fast effective scan rates, good discrimination against background and capacitive currents, and high sensitivity. In choosing the BIA parameters for performing square-wave voltammetry, it is important to ensure that the full voltammetric scan occurs during the injection period. This dictates the use of the lowest of the three possible flow rates for which an injection of $100 \mu \mathrm{L}$ lasts slightly more than $4 \mathrm{~s}$. Thus, the effective scan rate (defined as squarewave frequency multiplied by scan increment) must be chosen accordingly-for example, for a scan of $0.5 \mathrm{~V}$ width, this must be at least $125 \mathrm{mV} \mathrm{s}^{-1}$ assuming complete synchronization between voltammetric scan and injection.

A typical square-wave voltammogram recorded at $100-\mathrm{Hz}$ frequency during the injection of $100 \mu \mathrm{L}$ of ferrocyanide analyte is shown in Figure 8. Although discrimination against background currents is good, a background-subtracted squarewave voltammogram is better. In this case, the background 


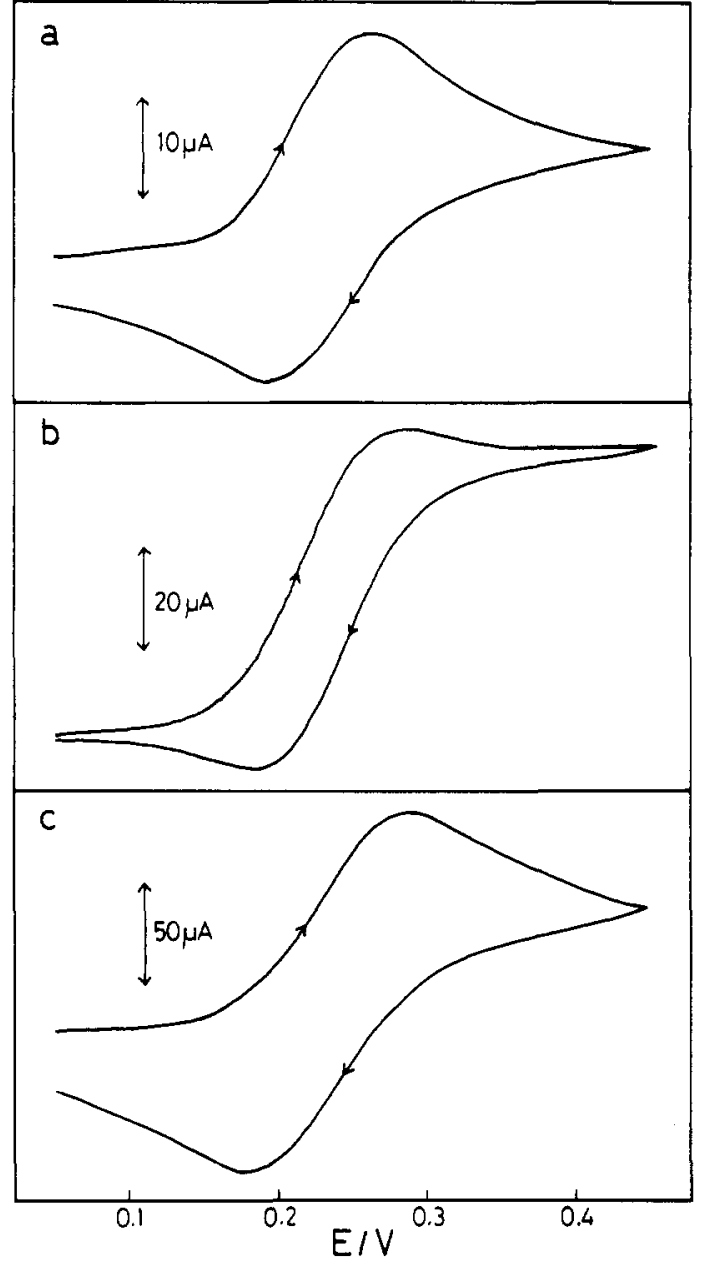

Flaure 7. Cyclic voltammograms for oxidation of $2.0 \mathrm{mM}$ ferrocyanide, $100-\mu \mathrm{L}$ injectlon. (a) After Injection; scan rate, $100 \mathrm{mV} \mathrm{s}^{-1}$. (b) During injection at flow rate of $24.5 \mu \mathrm{L} \mathrm{s}^{-1}$; scan rate, $250 \mathrm{mV} \mathrm{s}^{-1}$. (c) During injection at flow rate of $24.5 \mu \mathrm{L} \mathrm{s}^{-1}$; scan rate, $5.0 \mathrm{~V} \mathrm{~s}^{-1}$.

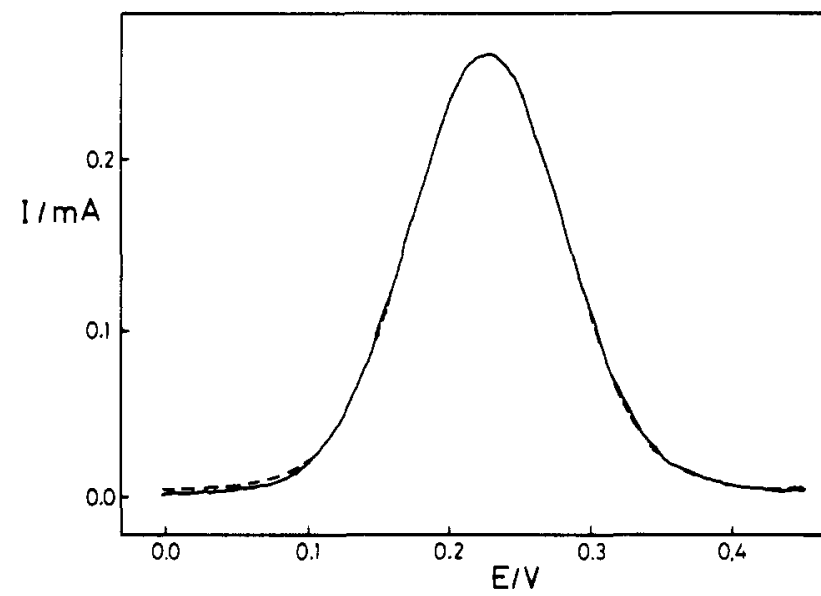

Flgure 8. Background-subtracted square-wave voltammogram recorded during injection of $100 \mu \mathrm{L}$ of $2.0 \mathrm{mM}$ potassium ferrocyanide at the low flow rate of $24.5 \mu \mathrm{L} \mathrm{s} \mathrm{s}^{-1}$. Square-wave parameters: frequency, $100 \mathrm{~Hz}$; amplitude, $50 \mathrm{mV}$; scan increment, $2 \mathrm{mV}$. Dotted line shows optimized fitting for a reversible $1 \theta^{-}$oxidation using the COOL algorithm.

voltammogram corresponded to the injection of a blank solution of $0.4 \mathrm{M} \mathrm{K}_{2} \mathrm{SO}_{4}$ electrolyte under exactly the same experimental conditions. Comparison with a voltammogram recorded in blank solution without injection showed that there is a small hydrodynamic component. After background subtraction, the peak is symmetrical, and the peak current corresponds to the half-wave potential which can be determined as $228 \mathrm{mV}$ vs SCE using curve optimization from the COOL algorithm as shown. The advantage of this approach as compared to the linear sweep method is that a continuous curve of the entire voltammetric wave is recorded, which should lead to higher precision and greater accuracy in the determination of the relevant parameters.

Variation of the square-wave amplitude and frequency were tested, giving the expected linear variation with the square root of frequency. This, together with synchronization criteria, led to the optimized parameters of $100-\mathrm{Hz}$ frequency and 50-mV amplitude for ferrocyanide oxidation. Calibration plots were constructed using background-subtracted square-wave voltammograms in the concentration range of $10^{-5}-10^{-3} \mathrm{M}$ using the following parameters: frequency, $100 \mathrm{~Hz}$; squarewave amplitude, $50 \mathrm{mV}$; scan increment, $2 \mathrm{mV}$. The slope of the linear plot was $0.109 \pm 0.001 \mu \mathrm{A} / \mu \mathrm{M}$, with a zero intercept. Comparison with the measurement of maximum limiting current in fixed-potential amperometric detection shows sensitivity enhancement by a factor of approximately 4 . The precision is greater, and the detection limit is lower at around $5 \mu \mathrm{M}$.

Square-wave scans of ascorbic acid oxidation lead to more drawn-out waves dependent on frequency, and splitting into two waves can occur depending on square-wave frequency. As in the analysis of the steady-state voltammetric curve, this shows the more complex nature of the oxidation process on platinum electrodes, which, as mentioned above, is the object of further study at the present time.

Comparison between BIA/Voltammetry, FIA/Voltammetry, and Microelectrodes. It is instructive to compare the advantages of voltammetry coupled with BIA and voltammetry coupled with flow injection analysis (FIA). A comparison of the general advantages of BIA with respect to FIA has already appeared, ${ }^{13}$ which points out the experimental simplification resulting from the removal of the carrier stream and continuous flow necessary for FIA. In most other aspects the techniques are similar, particularly when the sample dispersion is low in FIA. Several publications have appeared devoted to FIA/ voltammetry, including combination with cyclic voltammetry, ${ }^{14}$ with differential pulse voltammetry, ${ }^{15}$ and with squarewave voltammetry. ${ }^{16}$ However, in all the cited examples, injection volumes larger than the $100 \mu \mathrm{L}$ employed in this study are required; the lower sample volume required by BIA is an additional advantage.

An alternative for analyzing small volumes of solution is microelectrodes, ${ }^{17}$ they can sometimes reach lower detection limits but require more sample solution due to the necessity of placing the reference and auxiliary electrodes within the measurement cell. Thus, there are potential advantages relative to using microelectrodes in similar experimental conditions where reference and auxiliary electrodes need to be contained within the sample solution.

(13) Wang, J. Microchem. J. 1992, 45, 219-24.

(14) Cañete, F.; Rios, A.; Luque de Castro, M. D.; Valcárcel, M. Anal. Chim. Acta $1988,211,287-92$.

(15) Wang, J.; Dewald, H. D. Anal. Chim. Acta 1983, 153, 325-30.

(16) Wechter, C.: Sleszynski, N.; O’Dea, J. J.; Osteryoung, J. Anal. Chim. Acta $1985,175,45-53$.

(17) Montenegro, M. I., Queirós, M. A., Daschbach, J. L., Eds. Microelectrodes: theory and applications; Kluwer: Dordrecht, 1991. 


\section{CONCLUSION}

The potentialities of batch injection analysis for voltammetric as well as amperometric detection have been demonstrated. It has been shown that small injection volumes lead to greater electrolysis efficiencies, which may be useful for increasing the method's sensitivity. Linear sweep and cyclic and square-wave voltammetry combined with BIA allow thermodynamic and kinetic parameters of the electrode reaction to be obtained together with concentration determination. Of these, square-wave voltammetry, involving only one injection, is recommended as it provides a continuous voltammetric curve for posterior data treatment and curve fitting. It also leads to an increase in sensitivity by a factor of 4 relative to amperometric detection. This can be achieved with solution samples of only $100 \mu \mathrm{L}$ volume.

\section{ACKNOWLEDGMENT}

L.C.-M. wishes to thank the Tempus programme of the European Community (JEP Contract 4223-92/1) for a study grant.

Received for review March 8, 1994. Accepted June 6, $1994 .^{\circledR}$

- Abstract published in Advance ACS Abstracts, July 15, 1994. 